\title{
Erratum to: Abstracts from the ICBM 2014 Meeting
}

Published online: 24 March 2015

(C) International Society of Behavioral Medicine 2015

Erratum to: Int. J. Behav. Med. (2014) 21 (Suppl 1):S1-S216 DOI 10.1007/s12529-014-9418-2

Two abstracts were missing from the supplement. They are published here.

15 - Diabetes I - Lifestyle interventions in obesity and diabetes

Oral presentation

WEB-BASED CBT FOR PATIENTS WITH EATING DISORDERS: PRELIMINARY RESULTS OF A RANDOMIZED CONTROLLED TRIAL

E.D. ter Huurne $^{1}$, H.A. de Haan ${ }^{1}$, M.G. Postel ${ }^{1}$, C.A.J. de Jong ${ }^{2}$ ${ }^{1}$ Tactus Addiction Treatment, ENSCHEDE, Nederland

${ }^{2}$ Nijmegen Institute for Scientist Practitioners in Addiction, NIJMEGEN, Nederland

Introduction: In order to treat more patients with eating disorders in the Netherlands, a web-based CBT using intensive therapeutic support was developed. In this RCT we examined the efficacy of the web-based CBT.

Methods: Female patients with bulimia nervosa (BN), binge eating disorder (BED) and eating disorders not otherwise specified (EDNOS) were randomly assigned to the webbased CBT ( $\mathrm{n}=108)$ or waiting list control group (WL; $\mathrm{n}=106$ ). The web-based CBT included a structured online treatment using intensive therapeutic support. Participants communicated with their therapist asynchronously via the Internet twice a week. Participants in the WL received noreply email messages once every two weeks during the

The online version of the original article can be found at: http://dx.doi. org/10.1007/s12529-014-9418-2 waiting period of 15 weeks. Primary outcome measure was improvement in eating disorder psychopathology.

Results: Adherence data showed that more than half of the participants completed the web-based CBT with several significant differences between completers and non-completers. Study attrition was low with less than $10 \%$ losses to post-test. Participants of the web-based CBT and WL both showed improvements over time on eating disorder psychopathology, however, participants of the web-based CBT improved more. Secondary outcome data also showed more improvement in the web-based CBT compared to the WL for body dissatisfaction, physical and mental health, self-esteem, and social problems. For BMI no effects were found. Within the subgroups BN, BED, and EDNOS also several improvements were found on eating disorder related outcome measures.

Conclusion: Web-based CBT is effective in improving eating disorder psychopathology, body dissatisfaction and related health.

43 - Patients' perceptions of illness and treatment: targets for interventions to support medication adherence Symposium (speaker)

MODIFYING ADHERENCE-RELATED BELIEFS IN INFLAMMATORY BOWEL DISORDER: RANDOMIZED CONTROLLED TRIAL OF AN ONLINE INTERVENTION S.C.E. Chapman ${ }^{1}$, A. Sibelli ${ }^{2}$, P. Bondarek ${ }^{1}$, R. Horne ${ }^{1}$, A. Forbes $^{3}$, R. Driscoll ${ }^{3}$, A. Chater ${ }^{3}$

${ }^{1}$ UCL School of Pharmacy, LONDON, United Kingdom

${ }^{2}$ Kings College London, LONDON, United Kingdom, Nederland

Introduction: Nonadherence to maintenance medication is common in inflammatory bowel disease (IBD), increases flare-ups and healthcare costs, and is associated with doubts 
about need for maintenance medication and concerns about its potential adverse effects. Online intervention provides a potential cost-effective vehicle for the delivery of tailored interactive support to change these adherence-related beliefs.

Method: Participants with IBD taking azathioprine or mesalazine were recruited through a hospital clinic and patient website. An automated online questionnaire was used to 'profile' participants; those reporting doubts about need or concerns about adverse effects of medication were allocated to control $(n=176)$ and intervention groups $(n=153)$. Intervention participants received interactive exercises and persuasive messages tailored to their profiled concerns and doubts. The intervention's effect on beliefs, reported practical barriers and adherence was assessed at 1 and 3 months postintervention.
Results: Intervention participants had lower concerns about azathioprine than controls at 1 month follow-up, Intervention $\mathrm{m}=2.79$, Control $\mathrm{m}=3.03, \mathrm{t}(134)=2.16, \mathrm{p}=0.04$, and 3 months follow-up, Intervention $\mathrm{m}=2.67$, Control $\mathrm{m}=3.06, \mathrm{t}(81)=2.96$, $\mathrm{p}<0.01$. They also reported fewer practical barriers $(\mathrm{m}=2.18)$ than controls, $(\mathrm{m}=3.25)$ at 3 months. A higher proportion of the intervention group than controls reported high adherence at 3 months ( $41.7 \%$ vs. $20.5 \%)$. The groups did not differ on necessity beliefs.

Conclusions: Online interventions can be a useful tool for providing tailored support to patients with IBD at risk of nonadherence, and in particular concerns about medication. Future work will include interviews to assess the acceptability of the intervention, to inform development of online support services for IBD patients. 